\title{
Competitive Location and Pricing on Networks
}

\author{
Daniel Serra * \\ Dept. of Economics and Business \\ Universitat Pompeu Fabra \\ and
}

Charles ReVelle

Dept. of Geography and Environmental Engineering The Johns Hopkins University

\begin{abstract}
In this paper we consider a location and pricing model for a retail firm that wants to enter a spatial market where a competitor firm is already operating as a monopoly with several outlets. The entering firms seeks to determine the optimal uniform mill price and its servers' locations that maximizes profits given the reaction in price of the competitor firm to its entrance. A tabu seach procedure is presented to solve the model together with computational experience.
\end{abstract}

Key words: competitive location, discrete network location, spatial pricing Journal of Economic Literature classification: C61, R32,L81, R12

${ }^{*}$ Corresponding author: Daniel Serra, Department of Economics and Business, Universitat Pompeu Fabra, Trias Fargas 25-27, Barcelona 08005, Spain. Tel: 34-35421666, Fax: 34-3-5421746, e-mail: serra@upf.es 


\section{Introduction}

Hotelling's work on two firms competing in a linear market with consumers distributed uniformly along the line (also known as the ice-cream vendor problem) set the foundations of what is today the burgeoning field of competitive location. Competitive location is not only important from a practical point of view. It is also intriguing because, as Eiselt and Laporte (1989) point out, by modifying the original assumptions of the model the results obtained may differ significantly from Hotelling's conclusions: "relatively small changes in the model assumptions result in dramatic changes in the outcome" (p.237).

During the late thirties and early forties, several papers using the same spatial representation as Hotelling but modifying some of the economic assumptions appeared in the economic literature (Hoover (1936), Lerner and Singer (1937), and Smithies (1941)). There followed several decades of stagnation in the contribution of new insights in the field of competitive location in linear markets. Since the late seventies however, a myriad of different models have appeared in the literature of spatial economics and industrial organization. Useful reviews can be found in Ponsard (1983), Graitson (1987), Gabszewicz and Thisse (1991), and Eiselt and Laporte(1989).

Much of the effort in the new evolving literature has aimed at developing insights concerning the equilibrium pattern of competitive location and pricing. Nevertheless, "although these models (linear models) are rich in their 
theoretical insights about spatial competition and have greatly enhanced our understanding of locational interdependence, they provide very little guidance for developing practical approaches to facility location in competitive environments." (Ghosh and Craig (1984)).

Parallel to the development of this body of literature, a new field on location modeling was growing in the late sixties and seventies at a fast rate, namely facility location analysis. This field of research, coming basically from the fields of operations research, regional science and geography, dealt with the problem of locating new facilities in a spatial market in order to optimize one or several geographical and/or economic criteria. These criteria included overall distance minimization and transport and manufacturing cost minimization. The literature in facility location analysis is extensive: good sources of references can be found in Domschke and Drexl (1985), who identified over 1500 references on the subject, Love et al. (1988), and Chhajed et al. (1993). Although most of these models used more realistic spatial representations than the line, such as networks and planes, most of them dealt exclusively with non-competitive situations, and little attention was paid to the characterization of market equilibria.

From the late seventies, considerations on the interaction between competing facilities in discrete space have been developed following several different approaches. An extensive bibliographic survey with over 100 citations on competitive location can be found in Eiselt et al. (1993). 
One of the first of the questions that is addressed by several authors is whether or not a set of locations in the vertices of a network exist that will ensure a Nash equilibrium, that is, a position where neither firms have incentives to move. Wendell and McKelvey (1981) considered the location of two competitive firms with one server each and tried to find a situation where a firm would capture at least $50 \%$ of the market regardless of the location of its competitor. Results showed that there was not a general strategy for the firm that would ensure this capture if locations were restricted to vertices of the network. They did not develop a generic algorithm for finding solutions, but they looked at the possible locational strategies. They also examined the problem in a tree. Hakimi (1986) also analyzed extensively the problem of competitive location on vertices and proved that, under certain mathematical conditions such as concave transportation costs functions, that there exists a set of optimal locations on the vertices of the network.

A similar problem was studied by Lederer and Thisse (1990). Their problem not only looked at the specification of a site but also at the setting of a delivered price. They formulated the problem as a two-stage game, where in the first stage both firms choose locations and in the second stage they simultaneously set delivery prices schedules, and the result is that there is sub-game perfect Nash equilibrium. As Hakimi did, they also proved that if firm's transport costs are strictly concave, then the set of locational choices of the firm is reduced to the vertices of the network. As a consequence, the 
location problem can be reduced to a 2 -median problem if social costs are minimized.

A similar result was obtained by Labbe and Hakimi (1991). They developed a two-stage game in which two firms with one server each first select their location and then the quantities they will offer to each market. They proved that a sub-game perfect Nash equilibrium exists and that the locations occur on the vertices if transport costs are concave.

The problem of two firms competing in a spatial market has also been studied in the case where the market is represented by a tree. Eiselt (1992) proved that in such a case there is not a sub-game perfect Nash equilibrium if both prices and locations are to be determined. Eiselt and Laporte (1993) extended the problem to the location of 3 facilities in a tree. They found that the existence of equilibria depended on the distribution of weights. In both models, firms were allowed to locate on the edges of the network.

The game-theoretical models presented so far restrict themselves to the location of firms with one facility each that compete against each other. Tobin and Friesz (1986) examined the case of a profit-maximizing firm that entered a market with several plants. They considered price and production effects on the market, since the increase in the overall production level from the opening of new plants in a spatial market stimulates reactions in the competitors. These reactions might affect not only production levels, but also prices and locations. 
Tobin and Friesz developed two models: (1) a spatial price equilibrium model which determines equilibria in prices and production levels for a given number of firms, and (2) a Cournot-Nash oligopolistic model in which a few profit maximizing firms compete in spatially separated markets. They used both models to analyze the case of an entering firm that is going to open several new plants in spatially separated markets, and knows that its policy will have impact on market prices. Since profits depend on location and pricelevels and these depend on the reaction of the competitors, it is not possible to use a standard plant location model. To tackle the problem, they used sensitivity analysis on variational inequalities to relate changes in production to changes in price to obtain optimal locations. The model was solved using a heuristic procedure where in the first step a spatial competitive equilibrium model was obtained and, in the second step, a sensitivity analysis of profit to production changes was done using an integer non-linear program to select locations and production levels likely to maximize total profits. This model was generalized by Friesz et al. (1989) to allow the entering firm to determine not only production levels and the sites of its plants, but also its shipping patterns, and to examine different market strategies that can occur in the market (Miller et al. (1991)). Due to the mathematical complexity of these models, Miller et al. (1992) developed several heuristic methods to tackle the problem using the approach of variational inequalities (see, among others, Harker (1984) and Nagurney (1993)). 
Price-location modeling has been studied in a non-competitive model by Hanjoul et al. (1990). They develop three uncapacitated plant location models where different alternative spatial price policies are considered (uniform mill and delivered pricing, spatial discriminatory pricing). The models were solved using a heuristic procedure that combined price and location settings.

Another body of literature on competitive location deals with the siting of retail convenience stores. This type of store is characterized by (1) a limited and very similar product offering across outlets, (2) similar store image across firms, and (3) similar prices. Therefore, location is a major determinant of success.

Ghosh and Craig (1984) considered the location of several retail facilities by two servers. The problem is to locate retail facilities in a competitive market knowing that a competing firm will also enter this market. They used a minimax approach, where the entering firm maximizes its profit given the best location of the competitor. Potential locations were restricted to the vertices of the network. The firm's objective is to maximize the net present value of its investment over a long-term planning horizon. The model did not allow location at the same site for both firms and did not examine the issue of ties. Ghosh and Craig used a heuristic algorithm to obtain solutions. The algorithm is as follows: for each possible set of locations of firm $\mathrm{A}$, the best siting strategy is found for firm B. The final result is the set of locations where Firm A's objective is maximum given the best reactive location strategy of its 
competitor. A Teitz and Bart hill-climbing heuristic was used to determine the sites for both firms. The model is adapted to examine other strategies such as preemption, i.e., the identification of locations that are robust against competitive action. Other modifications included the relaxation of the number of stores that could be opened by each firm, and collusion by both servers.

In a similar model, Dobson and Karmarkar (1987) introduced the notion of stability in the location of retail outlets by two profit maximizing firms in a competitive market. Several integer programming models were developed to identify stable locations such that no competitors can enter the market and have profits given some rules on the competitive strategies. The models were solved using enumeration algorithms.

Most competitive location models assume that consumers patronize the closest shop. Karkazis (1989) considered two criteria that customers may use to decide which shop to patronize: a level criterion based on the preferences of a customer on the size of the facility and a distance criterion based on closeness to the store. He developed a model that would determine the location and number of servers to enter the market when there are other firms already operating in the market by maximizing the profit subject to a budget constraint. The problem was solved in a dynamic fashion since there is a trade-off between both criteria.

Another model that examines competition among retail stores in a spatial market was developed by ReVelle (1986). The Maximum Capture Problem 
(MAXCAP) has formed the foundation of a series of models. These models include issues such as the strategies that competing firms may adopt or the uncertainty that characterizes some situations. The MAXCAP model, based on the classical Maximal Location Covering Problem of Church and ReVelle (1974), consists of the location of servers by an entering firm so as to maximize its market share capture in a market in which competitor servers are already in position. Its integer linear formulation together with the maximum client capture objective has been the starting point for several location problems. Eiselt and Laporte (1989) modified the MAXCAP formulation to include attraction parameters based on gravity models and Voronoi diagrams. ReVelle and Serra (1991) extended the formulation to allow relocation of existing servers as well as the location of new servers. The MAXCAP model has also been adapted to consider facilities that are hierarchical in nature and where there is competition at each level of the hierarchy (Serra et al. (1992)). Another extension of the MAXCAP problem deals with the issue of a location and allocation game for two competitor firms, A and B, that each seek to locate $p$ facilities in a network: Firm A wants to locate its $p$ facilities so that B, which enters also with $p$ facilities after Firm A has located its facilities, will capture the minimum market value possible. That is, Firm A wishes to pre-empt Firm B in its bid to capture market share to the maximum extent possible (Serra and ReVelle (1994)). This preemptive model also has been adapted to the situation where Firm A does not even know how many new 
servers Firm B will locate (Serra and ReVelle (1995)). Another modification of the MAXCAP problem considered scenarios with different demands and/or competitor locations (Serra and ReVelle (1996)).

In this paper a new competitive model addresses the issue of location of several retail outlets by a firm and the fixing of a price in order to maximize profits, given the presence of a competitor firm. First, in section 2 the formulation model will be presented. In section 3 a heuristic method to solve it will be proposed. Computational experience and an example will be developed. Finally, some remarks and conclusions will be offered.

\section{The Maximum Capture Problem with Prices}

The original Maximum Capture Problem (MAXCAP) sought the location of a fixed number of outlets belonging to a firm in a spatial market with outlets from other firms already competing for clients. Competition was exclusively based on distance: a market was "captured" by a given server if there was no other server closer to it. If two servers from competing firms had a local market at the same distance, then they divided in equal part the capture of that market, as in a Hotelling's game. The objective of the entering firm was to maximize its market capture. This objective, given the assumptions on the characteristics of the retail stores, was almost equivalent to maximizing profits (Hansen et al. (1987)). A very similar problem was stated by Hakimi (1986). The main difference in the assumptions is that Hakimi did not allow 
half capture. In case of equal distances to the outlets from a node, the demand was fully allocated to the existing firm. In this paper we will use Hakimi's assumptions.

Now consider a spatial market where there is an existing firm (from now on, Firm B) operating with $q$ outlets. A new firm (Firm A) wishes to enter with $p$ servers and compete with firm $\mathrm{B}$ on the basis of price and locations. Both firms are profit maximizing. The product sold in this industry is homogeneous, in the sense that it is difficult to differentiate it among stores belonging to different firms. The space is discrete and is defined by a connected graph. On each vertex of the graph there is a local market with a given number of consumers that generates a demand for the product. Each consumer buys only one unit of the good in the "cheapest" outlet, where cheapest is defined momentarily.

Let $i$ and $I$ be the subindex and the set of local markets that are located on the vertices of the graph. Outlets are allowed to locate only on the vertices of the graph. Define $j$ and $J$ as the subindex and set of potential locations for Firm A's outlets, and $J^{B}$ the set of actual locations of the outlets of Firm B. Both firms follow a uniform mill pricing policy, so customers bear transportation costs. Therefore, the consumer's decision on patronizing a store is based on transportation costs and price. Consumers always go the outlet with the lowest total price, regardless of its ownership.

Let $d_{i j}$ be the network distance between local market $i$ and an outlet in 
$j$, and $t$ the unit transport cost. Both firms charge a mill price $p^{A}$ and $p^{B}$ to its customers irrespective of their location. We assume that prices do not change across outlets. Therefore, the price $\pi_{i}$ faced by consumers in each local market $i$ can be written as $\pi_{i}=p+t d_{i b_{i}}$, where $b_{i}$ is the closest outlet to $i$. The demand of local market $i$ is a function of the market's characteristics and the price it faces and is denoted as $D_{i}\left(\pi_{i}\right)$. Let $D_{i}\left(p+t d_{i b_{i}}\right)$ be the demand for the local market $i$, shopping at its closest outlet. Therefore, the demand function for each local market $i$ for Firm $\mathrm{A}$ is defined as follows: if $b_{i}^{A}$ is the closest Firm A's server to $i$ and $b_{i}^{B}$ the closest Firm B's server, then:

$$
D_{i}^{A}\left(\pi^{A}, \pi^{B}\right)=\left\{\begin{array}{lll}
D_{i}\left(\pi^{A}\right) & \text { if } & p^{A}+t d_{i b_{i}^{A}}<p^{B}+t d_{i b_{i}^{B}} \\
0 & \text { if } & p^{A}+t d_{i b_{i}^{A}} \geq p^{B}+t d_{i b_{i}^{B}}
\end{array}\right.
$$

that is, Firm A will capture the demand of local market $i$ if the total price $\pi_{i}^{A}$ (mill price plus transport costs to the closest A outlet) faced by consumers is lower than the total price $\pi_{i}^{B}$ reflecting the price set by the competitor firm and transport cost.

Assume that production entails for each outlet a fixed set-up cost that varies with location, $f_{j}$, and a constant marginal cost, $v$, so that for each outlet costs are given by $f_{j}+v q$, where $q$ is the amount of production at the site. If $J^{A}$ is the set of actual outlet locations $\left(J^{A} \subset J\right)$, then profits for firm A can be written as follows:

$$
\Pi^{A}=\left(p^{A}-v\right) \sum_{i \in I} D_{i}^{A}\left(\pi^{A}, \pi^{B}\right)-\sum_{j \in J^{A}} f_{j}
$$


The problem for firm A consists of determining the sites out of locations set $J^{A}$ and the price $p^{A}$ that maximizes profits. These profits also depend on other model parameters such as the location and prices of the competitor firm and the demand function of consumers among others.

If the demand function of the local markets is totally inelastic with respect to prices, it can be written as $D_{i}=a_{i}$, where $a_{i}$ is the total quantity that market $i$ will purchase, then the PMAXCAP problem can be stated as follows:

$$
\operatorname{Max} \Pi=\left(p^{A}-v\right)\left(\sum_{i \in I} a_{i} y_{i}\right)-\sum_{j \in J} f_{j} x_{j}
$$

subject to:

$$
\begin{aligned}
y_{i}^{A} \leq \sum_{j \in N_{i}\left(b_{i}^{B}\right)} x_{j}^{A} \quad \forall i \in I \\
\sum_{j=1}^{n} x_{j}^{A}=n^{A} \\
y_{i}^{A}, x_{j}^{A}=(0,1) \quad \forall i \in I, \forall j \in J
\end{aligned}
$$

where additional notation is:

$$
\begin{aligned}
N_{i}\left(b_{i}^{B}\right) & =\left\{\forall j \in J, \quad p^{A}+t d_{i j}<p^{B}+t d_{i b_{i}^{B}}\right\} \\
n^{A} & =\text { number of Firm A outlet servers. }
\end{aligned}
$$

and variables are defined as follows:

$$
\begin{aligned}
& y_{i}^{A}=\left\{\begin{array}{l}
1, \text { if Firm } A \text { captures demand node } i \\
0, \text { otherwise }
\end{array}\right. \\
& x_{j}^{A}=\left\{\begin{array}{l}
1, \text { if firm } A \text { locates a server at node } j \\
0, \text { otherwise }
\end{array}\right.
\end{aligned}
$$


where PMAXCAP is the problem which maximizes profit capture as opposed to population captured.

The first set of constraints (4) depends on the set $N_{i}\left(b_{i}^{B}\right)$, which is known a priori. Each one of the demand nodes $i$ has an associated set $N_{i}\left(b_{i}^{B}\right)$ which contains all the potential nodes at which Firm $A$ can locate a server and capture the demand of local market $i$. Therefore, if one of the variables $x_{j}^{A}$ belonging to the corresponding constraint is equal to 1 (i.e., a facility is located within the capture area of node $i$ ), then capture variable $y_{i}^{A}$ is allowed to be 1, which indicates that node $i$ has been captured by Firm $A$. Finally, the second constraint sets the number of servers that Firm $A$ is going to locate.

The objective defines the total profits that Firm $A$ can achieve with the siting of its $n^{A}$ servers. For each local market, there is demand $a_{i}$ to be captured. If $y_{i}^{A}=1$, then $\left(p^{A}-v\right) a_{i}$ is added to the revenues. Fixed costs are multiplied by $x_{j}$, so if an outlet opens in $j$, its associated fixed cost is substracted from the objective.

Observe that the last constraint (that limits the number of outlets to be located by Firm A) can be eliminated if fixed or opening costs are considered $\left(f_{j}>0\right)$. If profits are maximized, two forces act in opposite directions: increasing revenues by opening more outlets closer to the demand, but that in turn increases costs.

The basic difference between the MAXCAP problem and the PMAXCAP problem formulation presented relies (a) on the sets $N_{i}$ in restriction (1) re- 
spectively, (b) in the objective function, and (c) in the lack of any consideration of potential ties in capture (i.e., equal delivered prices). Eliminating a consideration of ties is reasonable because the likehood of ties in price + transport cots is very small.

The $N_{i}$ set contains all candidate nodes where consumers in local market $i$ would purchase from firm A, since the final price $\pi^{A}$ faced by them is lower than the final price of the closest competitor outlet $b_{i}^{B}$. While in the MAXCAP problem these sets were known a priori, in the PMAXCAP model this is not so since $p^{A}$ is not known, and therefore constraints (1) cannot be written extensively. On the other hand, the objective function is nonlinear, since variables $p$ and $y_{i}$ are unkown.

If the demand function is not completely inelastic, then the PMAXCAP problem has to reformulated using a P-median-like approach (PMAXMED):

$$
\max \Pi=\left(p^{A}-v\right) \sum_{i \in I} \sum_{j \in J} D_{i}^{A}\left(\pi^{A}, \pi^{B}\right) x_{i j}-\sum_{j \in J} f_{j} z_{j}
$$

subject to:

$$
\begin{array}{rlr}
\sum_{j \in J} x_{i j}=1 & \forall i \in I \\
x_{i j} \leq z_{j} \quad \forall i \in I, \forall j \in J \\
\sum_{j \in J} z_{j}=n^{A} & \\
x_{i j}, z_{j}=(0,1) \quad \forall i \in I, \forall j \in J
\end{array}
$$


where $x_{i j}$ is 1 if demand area $i$ is assigned to an outlet belonging to Firm A and $z_{j}$ indicates that there is a facility at node $j$ when its value is equal to 1 ; otherwise, its value is set to 0 . Constraint (7) assigns each demand area $i$ to only one outlet. For the assignment to be possible, $j$ needs to have an outlet. Constraint (8) controls this feature: $i$ cannot assign to $j$ unless there is an outlet at $j$. The last constraint (9) limits the number of servers that firm A will locate.

Again, this formulation of the PMAXCAP problem cannot be solved using standard linear programming relaxation and branch and bound when needed since (1) the objective is non linear, and (2) local demand functions $D_{i}^{A}\left(\pi^{A}, \pi^{B}\right)$ depend on the price $p^{A}$, which is unknown a priori.

In the following section a solution method for both formulations is proposed.

\section{A Bi-Level Heuristic Procedure to solve the PMAXCAP Problem}

Both problems can be solved using a two stage procedure where first locations are found using the original MAXCAP problem and then optimal prices are computed. Nevertheless, as Hanjoul et al. (1990) note, this is not acceptable since outlets locations "must be chosen conditionally upon clients' demand which, in turn, depends on prices. Conversely, it is readily apparent that the firm's optimal price relies heavily on its location choices. Hence, separating 
location and price decisions leads to sub-optimality."

The bilevel heuristic proposed in this paper combines a one opt procedure to obtain locations with a search method to find prices. Basically, in each possible exchange of the location of an outlet the price that gives maximum profits for Firm A is computed.

\section{The Price Search Heuristic}

This heuristic searches the price that maximizes profits for a firm that has several fixed outlets given the locations and price of the competitor firm. Observe that, given the price and locations of the competitor firm, and given the locations of the entering firm's servers, profits as a function of its price may present local optima. Suppose that $p^{B}$ is the price set by the competitor firm and the entering firm fixes a price $p^{A}$, where profits are $\Pi^{A}=\Pi^{A}\left(p^{A}, p^{B}\right)$. If the entering firm now increases its price by a small amount $\epsilon, p^{A^{\prime}}=p^{A}+\epsilon$, two opposite effects may act on profits. For a given node $i$, where $p^{A}+t d_{i b_{i}^{A}}<$ $p^{B}+t d_{i b_{i}^{B}}($ i.e., $i$ is captured by Firm $A):$

- if $p^{A^{\prime}}+t d_{i b_{i}^{A}}<p^{B}+t d_{i b_{i}^{B}}$, node $i$ is still captured by the closest server $b_{i}^{A}$. Therefore, local profits at node $i$ will increase if $\left(p^{A}-v\right) D_{i}\left(\pi^{A}, \pi^{B}\right)<$ $\left(p^{A^{\prime}}-v\right) D_{i}\left(\pi^{A^{\prime}}, \pi^{B}\right)$ or, in other terms if the demand is inelastic. On the contrary, if $\left(p^{A}-v\right) D_{i}\left(\pi^{A}, \pi^{B}\right)>\left(p^{A^{\prime}}-v\right) D_{i}\left(\pi^{A^{\prime}}, \pi^{B}\right)$ or, if the demand is elastic, profits obtained from node $i$ will be reduced.

- On the other hand, if $p^{A^{\prime}}+t d_{i b_{i}^{A}}>p^{B}+t d_{i b_{i}^{B}}$, node $i$ is lost to the 
competitor with the corresponding decrease in Firm A's profits relative to demand node $i$.

Therefore, two effects act on Firm A's profits as prices $p^{A}$ is increased. On the one hand, profits may experience a reduction since (1) some nodes may be lost to the competitor because the price $\pi_{i}$ paid by consumers at the closest competitor facility becomes lower than the one paid at Firm A's; and (2) even if the node remains captured by the entering firm after the increase in prices, profits may be reduced since the increase in price does not compensate for the decrease in quantity demanded. On the other hand, local profits in a given demand node may increase since the increase in prices more than compensates for the reduction in quantity demanded at other nodes.

As the price $p^{A}$ increases, it may happen that some local optimum may appear. Therefore, the profit function may not be unimodal. In the computational experience this characteristic of the profit function dependent on price will be shown.

The heuristic to determine the price that maximizes profits for Firm A given sets $J^{A}$ (the location of A's outlets) and $J^{B}$ (the location of B's outlets), and given B's price $p^{B}$ proceeds as follows:

First, lower and upper bounds $p_{l}$ and $p_{u}$ for the price for Firm A are found. Then, a price $p_{c}$ within the range is chosen and profits are computed. Then $p_{c}$ is decreased by a small value $\epsilon$ and the new profit is computed. If it is better that the previous one, it is stored as the best current solution. If not, 
it is discarded. The procedure is done until the lower bound $p_{l}$ is reached or until the profits found are lower that a percentage tolerance level $\tau$ of the best profits found so far. Now it is necessary to do the same for the range $\left[p_{c}, p_{u}\right]$. The procedure starts again from the price found at the beginning and in each step the current price $p_{c}$ is increased by $\epsilon$, profits are computed and compared to the best solution found so far. The procedure stops when $p_{u}$ is reached or if the profits found are lower than $\tau$ times the best profits found.

The upper bound is found by computing the highest price that Firm A can set before having 0 revenues. This is computed as follows: for each local market $i$, find the consumer price to the closest Firm B outlet $\left(p^{B}+t d_{i b_{i}^{B}}\right)$ and the transportation costs to the closest Firm A outlet $\left(t d_{i b_{i}^{A}}\right)$. If $p_{A}=$

$p^{B}+t d_{i b_{i}^{B}}-t d_{i b_{i}^{A}}$ both firms divide the local market revenues. If $p_{A}$ is larger, all local revenues in $i$ will be lost to the competitor. Therefore:

$$
p_{u}=\max \left(p^{B}+t d_{i b_{i}^{B}}-t d_{i b_{i}^{A}}, i \in I\right)
$$

i.e., the level at which no one patronizes Firm A.

The lower bound is found by setting $p_{l}=v$.

A more formal description of the heuristic follows:

\section{Initial stage:}

Given a set of locations for Firm $\mathrm{A}$ and Firm B and $p^{B}$ Set $p_{l}^{A} \leftarrow v$. set $p_{u} \leftarrow \max \left(p^{B}+t d_{i b_{i}^{B}}-t d_{i b_{i}^{A}}, i \in I\right)$ Choose an initial price $p_{0}^{A}$ within the 
interval $\left[p_{l}, p_{u}\right]$ and compute initial profits $\Pi_{0}$ for Firm A.

\section{Iterative stage:}

Phase 1: Set $t \leftarrow 1$. Set $p_{t}^{A} \leftarrow p_{t-1}^{A}+\epsilon$ and compute $\Pi_{t}$. If $\Pi_{t}>\Pi_{t-1}$ then set $\hat{\Pi} \leftarrow \Pi_{t}$ and $\hat{p} \leftarrow p_{t}^{A}$ as the current best solution. Set $t \leftarrow t+1$ repeatedly until first stopping rule applies. Then start Phase 2.

Phase 2: Reset $t \leftarrow 1$. Set $p_{t}^{A} \leftarrow p_{t-1}^{A}-\epsilon$ and compute $\Pi_{t}$. If $\Pi_{t}>\Pi_{t-1}$ then set $\hat{\Pi} \leftarrow \Pi_{t}$ and $\hat{p} \leftarrow p_{t}^{A}$ as the current best solution. Set $t \leftarrow t+1$ until second stopping rule applies.

First stopping rule: $\Pi_{t}<\tau \hat{\Pi}$ or $p_{t}^{A}=p_{l}^{A}$.

Second stopping rule: $\Pi_{t}<\tau \hat{\Pi}$ or $p_{t}^{A}=p_{u}^{A}$.

So far, we have shown how to find the best value of $p^{A}$ given the prespecified locations of $\mathrm{A}$ and $\mathrm{B}$, and B's prices. Now we are ready to start the procedure to find optimal prices and locations.

\section{The Location Heuristic}

The PMAXCAP heuristic procedure is iterative, and the first iteration has two phases. In the first phase, Firm A locates $p$ servers using a greedy adding procedure, where in each iteration the vertex with the best profit level is added to the set of locations, until $n^{A}$ is reached.

In the second phase, Firm A will try to find a better solution by relocating one or more of the $n^{A}$ outlets using a vertex-substitution procedure. At each iteration, Firm A will relocate one of its servers and then use the price heuristic 
to obtain the optimal price and profits. If the relocation has provided a set of positions and prices that is better than before the one-opt trade, i.e., Firm A's profits are higher, it will keep the new set of locations as the best so far. Otherwise, Firm A will ignore the relocation and will restore the previous solution. The one-opt trade will be done for all nodes and Firm A servers and repeated until no cycle results in an improvement.

Since the second phase only considers vertices that improve the objective, the heuristic may end in a local optimum.

In order to avoid being trapped in a local optimum, a tabu search procedure is developed, similar to the one presented by Benati and Laporte (1994). In essence, this tabu search explores a part of the solution space by repeatedly examining all neighbors of the current solution, and moving to the best neighbor even if this causes the objective function to deteriorate. To avoid cycling, recently examined solutions are inserted in a constantly updated tabu list. The heuristic proceeds as follows:

\section{Price-Location Heuristic}

\section{PhASE 1}

1. Set $J_{0}^{A} \leftarrow \emptyset, p_{0}^{A} \leftarrow p^{B}$ and $i \leftarrow 1$.

2. Set $J_{k}^{A} \leftarrow J_{k-1}^{A} \cup v_{k}$, where $v_{k}$ represents index of the vertex with the largest increase in capture: 


$$
\max _{v_{k} \in V}\left[\Pi\left(J_{k-1}^{A} \cup v_{k}, p^{A}\right)-\Pi\left(J_{k-1}^{A}, p^{A}\right)\right]
$$

3. Set $k \leftarrow k+1$ and repeat step 2 until $k=n^{A}$.

Phase 2

1. Set $J^{A *} \leftarrow J_{n^{A}}^{A}, \hat{\Pi} \leftarrow \Pi\left(J_{n^{A}}^{A}, p^{A}\right)$ and $p^{A *} \leftarrow p^{A}$.

2. Set $t \leftarrow 0$ and $\Pi^{0} \leftarrow \hat{\Pi}$

3. Set $J_{t}^{A} \leftarrow J_{t-1}^{A}-v_{k}+v_{l}$, where $v_{k} \in J_{p}^{A}$ and $v_{l} \in\left(J-J_{t-1}^{A}\right)$.

4. If $\Pi\left(J_{t}^{A}, p_{t}^{A}\right)>\hat{\Pi}, \quad J^{A *} \leftarrow J_{t}^{A}, \hat{\pi} \leftarrow \Pi\left(J_{t}^{A}, p^{A}\right)$ and $p^{A *} \leftarrow p_{t}^{A}$. Repeat step 3 until all vertices and outlets have been exchanged.

5. If $\hat{\Pi}>\Pi\left(J_{0}^{A}, p_{0}^{A}\right)$, set $\Pi^{0} \leftarrow \hat{\Pi}, p_{0}^{A} \leftarrow p^{A *}$ and goto step 3 . If not, go to phase 3 .

\section{Phase 3}

1. Reset $t \leftarrow 0$.

2. Set $\Pi^{0} \leftarrow \hat{\Pi}$ and $p_{0}^{A} \leftarrow p^{A *}$. No vertex is tabu.

3. Consider all solutions $J_{t}^{A, i}$ of $J_{t}^{A}$ given by adjacent nodes, obtained exchanging an outlet from $v_{i}^{\prime} \in J_{t}^{A}$ to $v_{i}^{\prime \prime} \in J-J_{t}^{A}$. Relabel all $J_{t}^{A, i}$ solutions in decreasing order of $\Pi\left(J_{t}^{A, i}, p_{t}^{A, i}\right)$. Relabel all vertices accordingly. Set $i \leftarrow i+1$. 
4. If $\Pi\left(J_{t}^{A, i}, p_{t}^{A, i}\right)>\hat{\Pi}$ or if $v_{i}^{\prime \prime}$ is not tabu, set $J_{t+1}^{A} \leftarrow J_{t}^{A, i}, \hat{\Pi} \leftarrow \Pi\left(J_{t}^{A, i}\right)$, declare $v_{i}^{\prime}$ tabu until $t+\theta$, where $\theta$ is a pre-fixed value, and go to step 5. Otherwise, set $i \leftarrow i+1$. If $i$ is larger than the number of adjacent solutions, set $i$ equal to the index of the vertex $v_{i}^{\prime \prime}$ with the lowest tabu $\operatorname{tag} t+\theta$ and lift the tabu status of $v_{i}^{\prime \prime}$. Repeat step 3.

5. Set $t \leftarrow t+1$. If $t$ is less than a pre-fixed upper bound $T$, go to step 2 in phase 3. Otherwise, stop

The main difference between this heuristic and the one developed by Benati and Laporte is that their starting solution is obtained by a greedy adding procedure. In ours, the initial solution is obtained by a vertex substitution procedure, and therefore the initial solution will be near-optimal or even optimal (see, for example, Rosing and ReVelle 1997). After finishing the procedure, Benati and Laporte re-start it by using a diversification step: a broader exploration of the solution space is obtained by starting from the least visited vertices. In our heuristic, as it will be shown in the computational experience, this seems unnecessary.

So far it has been assumed that Firm B will not react to Firm A's entry and to the price that A chooses. This is unlikely to occur. Suppose that Firm A now knows that its competitor will react to its entry by changing prices so as to maximize profits given the locations of Firm A's outlets and its price. Now Firm A's objective is to find the optimal location and prices that will 
optimize A's profits given the reaction in prices by its competitor. In this sense, it is a leader-follower game where Firm A is the leader.

This problem can be solved as follows: given a set of locations for both firms, price reaction functions can be computed for each one of them. By reaction function it is meant what the best price is (the price that maximizes profits) given the price of the competitor. The reaction function of Firm A is such that $p^{A}=f^{A}\left(p^{B}\right)$, where for each $p^{B}$ there is a $p^{A}$ that maximizes $\Pi^{A}$. Similarly, there is a reaction function $f^{B}\left(p^{A}\right)$ for Firm B given $p^{A}$. Since reactions functions are known for both firms, firm A can use Firm B's reaction function to find the price that will maximize its profits given firm B's reaction. Firm A can use the price heuristic to determine its best policy. In each step of the iterative stage where $p_{t}^{A}$ is determined, Firm A can use the price heuristic for firm B to obtain the price that Firm B will set given $p_{t}^{A}$. Once $\hat{p}_{t}^{B}$ is determined, Firm A can compute profits $\Pi_{t}^{A}$. A will choose the $\hat{p}_{t}^{A}$ that will maximize its profits. More formally, the heuristic is as follows:

\section{Price Competitive heuristic}

Initial stage: Given a set of locations for both Firms and $p^{B}$, set $p_{l}^{A} \leftarrow v$. set $p_{u}^{A} \leftarrow \max \left(p^{B}+t d_{i b_{i}^{B}}-t d_{i b_{i}^{A}}, i \in I\right)$. Choose an initial price $p_{0}^{A}$ within the interval $\left[p_{l}^{A}, p_{u}^{A}\right]$. Compute the best price $\hat{p}_{0}^{B}$ for Firm $\mathrm{B}$ using the price heuristic given $p_{t}^{A}$. Obtain $\Pi_{t}^{A}\left(p_{0}^{A}, \hat{p}_{0}^{B}\right)$ 


\section{Iterative stage:}

Phase 1: Set $t \leftarrow 1$. Set $p_{t}^{A} \leftarrow p_{t-1}^{A}+\epsilon$. Compute the best price $\hat{p}_{t}^{B}$ for Firm $\mathrm{B}$ using the price heuristic given $p_{t}^{A}$, and then $\Pi_{t}^{A}\left(p_{t}^{A}, \hat{p}_{t}^{B}\right)$. If $\Pi_{t}^{A}\left(p_{t}^{A}, \hat{p}_{t}^{B}\right)>\Pi_{t}^{A}\left(p_{t-1}^{A}, \hat{p}_{t-1}^{B}\right)$ then set $\hat{\Pi}^{A} \leftarrow \Pi_{t}^{A}$ and $\hat{p}^{A} \leftarrow p_{t}^{A}$ as the current best solution. Set $t \leftarrow t+1$ until first stopping rule applies. Then start Phase 2.

Phase2: Reset $t \leftarrow 1$. Set $p_{t}^{A} \leftarrow p_{t-1}^{A}-\epsilon$. Compute the best price $\hat{p}_{t}^{B}$ for Firm $\mathrm{B}$ using the price heuristic given $p_{t}^{A}$, and then $\Pi_{t}^{A}\left(p_{t}^{A}, \hat{p}_{t}^{B}\right)$. If $\Pi_{t}^{A}\left(p_{t}^{A}, \hat{p}_{t}^{B}\right)>$ $\Pi_{t}^{A}\left(p_{t-1}^{A}, \hat{p}_{t-1}^{B}\right)$ then set $\hat{\Pi}^{A} \leftarrow \Pi_{t}^{A}$ and $\hat{p} A \leftarrow p_{t}^{A}$ as the current best solution. Set $t \leftarrow t+1$ until second stopping rule applies.

First stopping rule: $\Pi_{t}^{A}<\tau \hat{\Pi}^{A}$ or $p_{t}^{A}=p_{u}^{A}$.

Second stopping rule: $\Pi_{t}^{A}<\tau \hat{\Pi}^{A}$ or $p_{t}^{A}=p_{l}^{A}$.

Now the price heuristic used within the one-opt procedure can be replaced by the price-competitive heuristic in steps 2 and 4 . Firm A will evaluate each one-opt trade based on the best profits it can get given the price reaction of B. Firm A will choose the set of locations and the price that will maximize its profits given Firm B's price reaction.

By using the Competitive Price Location Heuristic (CPLH) Firm A can achieve a situation that will maximize its profits given the reaction of its competitor. Since for each $p^{A}$ the optimal Firm B's price $p^{B}$ is computed, at 
the end of the procedure prices $\bar{p}^{A}, \hat{p}^{B}$ and profits $\bar{\Pi}^{A}, \hat{\Pi}^{B}$ are known. Observe that $\Pi^{B}$ is the best profit that firm $B$ can achieve given its locations and the price and locations of Firm A.

\section{Computational Experience}

Computer programs have been written in FORTRAN 77 in order to solve the competitive price location problem described above. The program was run on 4 test problems generated in the following way. $15,30,50$ and 70 points were randomly chosen in a 100 by 100 square for each test problem. Each point is at the same time the location of a local market and the potential site for an outlet. Transportation costs between points are equal to Euclidean distances.

All weights associated to each vertex have been set equal to 100 . A linear demand function has been selected, and the slope $\alpha$ has been chosen between 0.5 and 3.0, depending on the number of vertices. The fixed and variable production costs were set to 0 . The tolerance level $\tau$ has been chosen equal to 0.8 and the price parameter $\epsilon$ was set to 0.5 .

For all generated networks, the locations of Firm B were obtained by using the price heuristic together with a vertex substitution (Teitz and Bart) heuristic, as if there were no competitors in the market. Each one-opt trade was valued using the price heuristic. In this sense the locations and price found for firm B correspond to the optimal or near-optimal monopolistic solution: Firm B maximizes profits by fixing prices and locations without competition. 
Therefore, in each scenario Firm B starts with a good positioning of its servers.

Once the initial locations for Firm B were obtained, the price-location heuristic was used to obtain the final solutions for Firm A, using the pricecompetitive heuristic to compute the objective value. That is, in all runs Firm A considered that Firm B would react in prices.

First, in order to test the efficiency of the heuristic algorithm, 100 15-node networks were generated for $\alpha=0.5,1$ and 1.5 respectively. For each network, optimal solutions were obtained by enumerating all locations for the entering firm and, for each set of locations, computing the competitive-price heuristic to obtain optimal prices. Results are presented in Table 1. Computing times are presented in Table 2.

The price-location heuristic achieved in most cases the optimal solution. The vertex substitution procedure (phase II) obtained at least in $75 \%$ of the runs the optimal solution. When phase II did not obtain the optimum, phase III achieved in at least $50 \%$ of the runs the optimal solution. In general, the price-location heuristic performed better in terms of optimal solutions found as the slope of the demand function increased. This is also the case for computing times.

\section{An Example}

In this section the PMAXCAP model is applied to a 55-node network (Swain 1974, see Table 4 and Figure 1). Four different scenarios are examined with 
regard to the number of outlets to be located and the slope of the demand function. Firm B is operating as a monopoly in the market with several outlets. The demand function in each local market is linear and the same, except for the intercept, that is equal to the weight of the vertices. These intercepts are shown in table 2 of the appendix.

The locations and the price for Firm B have been obtained using the method described in the last section for the monopoly situation. That is, as a monopolist, Firm B has a good positioning in the market. Unit transportation costs are linear with distance and equal to 1 . Fixed and variable costs are set to 0 .

Firm A knows that Firm $B$ will react in prices, so it will use the pricecompetitive heuristic in phase II and III to obtain what price it has to set to maximize profits given Firm B's reaction. Results are presented in Table III.

In the first column the number of outlets for Firm A and Firm B and the slope of the demand function are presented. In the second column the initial scenario Firm B as a profit maximizer monopolist is presented. Then, results found in the different phases of the algorithm are shown. In none of the four runs phase III of the algorithm improved the solution found in phase II.

As mentioned before, given $\bar{p}^{B}, J^{A}$ and $J^{B}$, profits as a function of prices, given the price of the competitor may present some local optima. These can be seen in Figure 2, where the profit function corresponds to Firm B's final solution where $n^{A}=3, n^{B}=3$ and $\alpha=0.3$, and where $p^{A}=65.0$. 


\section{Conclusions}

In this study, market entry by a firm with several outlets has been studied within a competitive locational framework. In a system where two firms that offer the same good or service seek to enter a market, the location of their servers together with the setting of prices play a dominant role in the final profits that can be achieved.

A duopoly model that seeks to locate servers and set mill prices to maximize the market capture by a firm has been presented. The model, based on the MAXCAP problem, is quite efficient in obtaining solutions, even though no exact algorithm has been found to obtain optimal locations and prices.

A feature of the heuristics presented is that there is no need to make assumptions on the demand functions, production and transportation cost functions. The CPLH method can be used with any non-linear specification of these functions, and regardless of convexity or concavity properties.

The models proposed specify the number of servers $p$ that firm A is going to locate. This assumption can be relaxed by applying the heuristic for $p=$ $1,2, \ldots, n$ and choosing $p$ as the one that gives higher profits.

As seen in the computational experience, the price heuristic can be used to find the locations and price of a firm that enters with several outlets in a spatial market without competition. This is an alternative method to the one proposed by Hanjoul et al. (1990) if $p$ is not specified. 
The CPLH heuristic can be applied to different competitive situations. For example, suppose that Firm B is operating in the market and knows that Firm A will enter to compete. Firm B might want to find what its best price is given the Firm A's entry. Therefore, Firm B can use the CPLH and the PLH heuristics as follows: In the iterative stage of the PLH heuristic, Firm $\mathrm{B}$ would use the CPLH heuristic to find, given its price, where Firm B will locate and what price it will set. Firm B will choose the price that maximizes its profit given the locations and price of Firm A. In this sense, Firm B will find a price that will try to pre-empt Firm A in its bid to capture market share to the maximum extent possible.

The problem that remains to be solved relies on the competitive behavior of both firms. Recall that, once the optimal solution is found, while Firm B has no incentive to change its price since it is the best one given the entrance of Firm $\mathrm{A}$ in the market, this one can improve its profit levels by using the price heuristic. This situation leads to a sequential price war, where in each stage firms alternatively find the price that maximizes profits given the price fixation of the competitor in the previous stage.

It may be the case that by entering a price war, both firms will never find a better profit level than the one achieved at the end of the price-location heuristic. If both firms know this fact (by applying the price-competitive heuristic), they may cooperate by not entering a price war. In this sense, it can be defined as a sort of cooperative equilibrium. 
On the other hand, since reaction functions are known for a given set of locations for both firms, non-cooperative equilibria may also be identified applying the standard approach used in Industrial Organization Theory. This can been seen in Figure 3. In this graphic the reaction functions in prices for both firms are depicted, corresponding to the final solution obtained in the last section with $n^{A}=3, n^{B}=3$ and $\alpha=0.3$. The intersections of both functions may imply non-cooperative equilibria, even though these may not be stable and some cycling could occur. Further research can be developed to try to examine cooperative and non-cooperative equilibria in networks using the modelling approach presented here.

Finally, in this paper, a uniform mill pricing policy has been considered. The model can be adapted to other forms of pricing, such as uniform delivered pricing and spatial discriminatory pricing. 


\section{$7 \quad$ Acknowledgements}

This research was partially done under DCIGYT grants PB95-0980 and PB95-

0978, Ministry of Education, Spain. The authors gratefully acknowledge the comments of Andreu Mas-Colell and Walter Garcia-Fontes on early versions, as well as the suggestions received from the participants in the Applied Economics Workshop at UPF. All remaining errors are of our own responsibility. 


\section{References}

Benati, S. And G. Laporte, 1994, "Tabu search algorithms for the $\left(r \mid x_{p}\right)$ medianoid and $(r \mid p)$ centroid problems," Location Science, 2(4):193-204.

Chhajed, D., R.L. Francis, and T.J. Lowe, 1993, "Contributions of operations research to location analysis," Location Science, 1(4):263-287.

Church, R. And C. ReVelle, 1974, "The maximal covering location problem," Papers of the Regional Science Association, 32:101-118.

Dobson, G. And U.S. Karmarkar, 1987, "Competitive location on a network," Operations Research, 35(4):565-574.

Domschke, W. And A. Drexl, 1985, "Location and layout planning: An international bibliography, " in Lecture Notes in Economics and Mathematical Systems, volume 238. (Springer, Berlin).

EISElT, H., 1992, "Hotelling's duopoly on a tree," Annals of Operations Research, 40:195-207.

Eiselt, H. AND G. Laporte, 1989, "Competitive spatial models," European Journal of Operational Research, 39:231-242.

1993, "The existence of equilibria in the 3 -facility hotelling model in a tree," Transportation Science, 27(1):39-43. 
Eiselt, H., G. Laporte, and J.F. Thisse, 1993, "Competitive location models: A framework and bibliography," Transportation Science, $27(1): 44-54$

Friesz, T., L. Tobin, And T. Miller, 1989, "Existence theory for spatially competitive network facility location models," Annals of Operations Research, 18:267-276.

Gabszewicz, J. AnD J.F. Thisse, 1991, "Location," in Auman, R. and S. Hart, editors, Handbook of Game Theory with Economic Applications. (North-Holland).

Ghosh, A. And C.S. Craig, 1984, "A location allocation model for facility planning in a competitive environment," Geographical Analysis, 16(1):3951.

Graitson, D., 1987, "Spatial competition a la hotelling: A selective survey," Journal of Industrial Economics, 31:13-25.

HAKimi, S., 1986, "p-median theorems for competitive location," Annals of Operations Research, 5:79-88.

Hanjoul, P., P. Hansen, D. Peeters, and JF. Thisse, 1990, “Uncapacitated plant location under alternative spatial policies," Management Science, 36(1):41-57. 
Hansen, P., M. Labbe, D. Peeters, , and J.F Thisse, 1987, "Facility location analysis," in Arnott, R., editor, Systems of Cities and Facility Location, pages 1-70, (Harwood Academic Publishers).

HARKER, P., 1984, “A variational inequality approach for the determination of oligopolistic market equilibrium," Mathematical Programming, 30:105111.

Hoover, E., 1936, "Spatial price discrimination," Review of Economic Studies, 4:182-191.

KARKAZIS, J., 1989, "Facilities location in a competitive environment: A promethee based multiple criteria analysis," European Journal of Operational Research, 42:294-304.

LABBE, M. AND S.L. HAKIMI, 1991, "Market and location equilibrium for two competitors," Operations Research, 39(5):749-756.

Lederer, P. AND J.F. Thisse, 1990, “Competitive location on networks under delivere pricing," Operations Research Letters, 9:147-153.

Lerner, A. And H.W. Singer, 1937, "Some notes on duopoly and spatial competition," Journal of Political Economy 39, pages 145-186.

Love, R., J.G. Morris, and G.O. Wesolovsky, 1988, Facilities Location: Models and Methods. (North Holland, New York). 
Miller, T., T.L. Friesz, And R.L. ToBin, 1992, "Heuristic algorithms for delivered price spatially competitive network facility location problems," Annals of Operations Research, 34:177-202.

Miller, T., R.L. Tobin, And T.L. Friez, 1991, "Stackelberg games on a network with cournot-nash oligopolistic competitors," Journal of Regional Science, 31:435-454.

Nagurney, A., 1993, Network Economics: A Variational Inequality Approach. (Kluwer Academic Publishers, Boston).

Ponsard, C., 1983, A History of Spatial Economic Theory. (Springer-Verlag, Berlin).

REVELLE, C., 1986, “The maximum capture or sphere of influence problem: Hotelling revisited on a network," Journal of Regional Science, 26(2):343357.

ReVelle, C. And D. Serra, 1991, "The maximum capture problem including relocation," Information and Operations Research, 29(2):130-138.

Rosing, K. And C. ReVelle, 1997, "The heuristic concentration for pmedian problems," Forthcoming in European Journal of Operational Research. 
Serra, D., V. Marianov, and C. ReVelle, 1992, “The hierarchical maximum capture problem," European Journal of Operational Research, $62(3)$

Serra, D. AND C. REVElle, 1994, "Market capture by two competitors: The preemptive capture problem," Journal of Regional Science, $34(4): 549-561$.

1995, “Competitive location in discrete space," in Drezner, Z., editor, Facility Location: A survey of methods and applications, Springer series in Operations Research, pages 367-386. (Springer, New York).

1996, "The maximum capture problem with uncertainty," Environment and Planning B, 62:49-59.

Smithies, A., 1941, "Optimum location in spatial competition," Journal of Political Economy, 49:423-439.

SWAIN, R., 1974, "A parametric decomposition algorithm for the solution of uncapacitated location problems," Management Science, 21:189-198.

Tobin, R. And T.L. Friesz, 1986, "Spatial competition facility location models: definition, formulation and solution approach," Annals of Operations Research, 6:49-74. 
Wendell, R. AND R.D. MCKelvey, 1981, "New perspectives in competitive location theory," European Journal of Operational Research, 6:174182. 


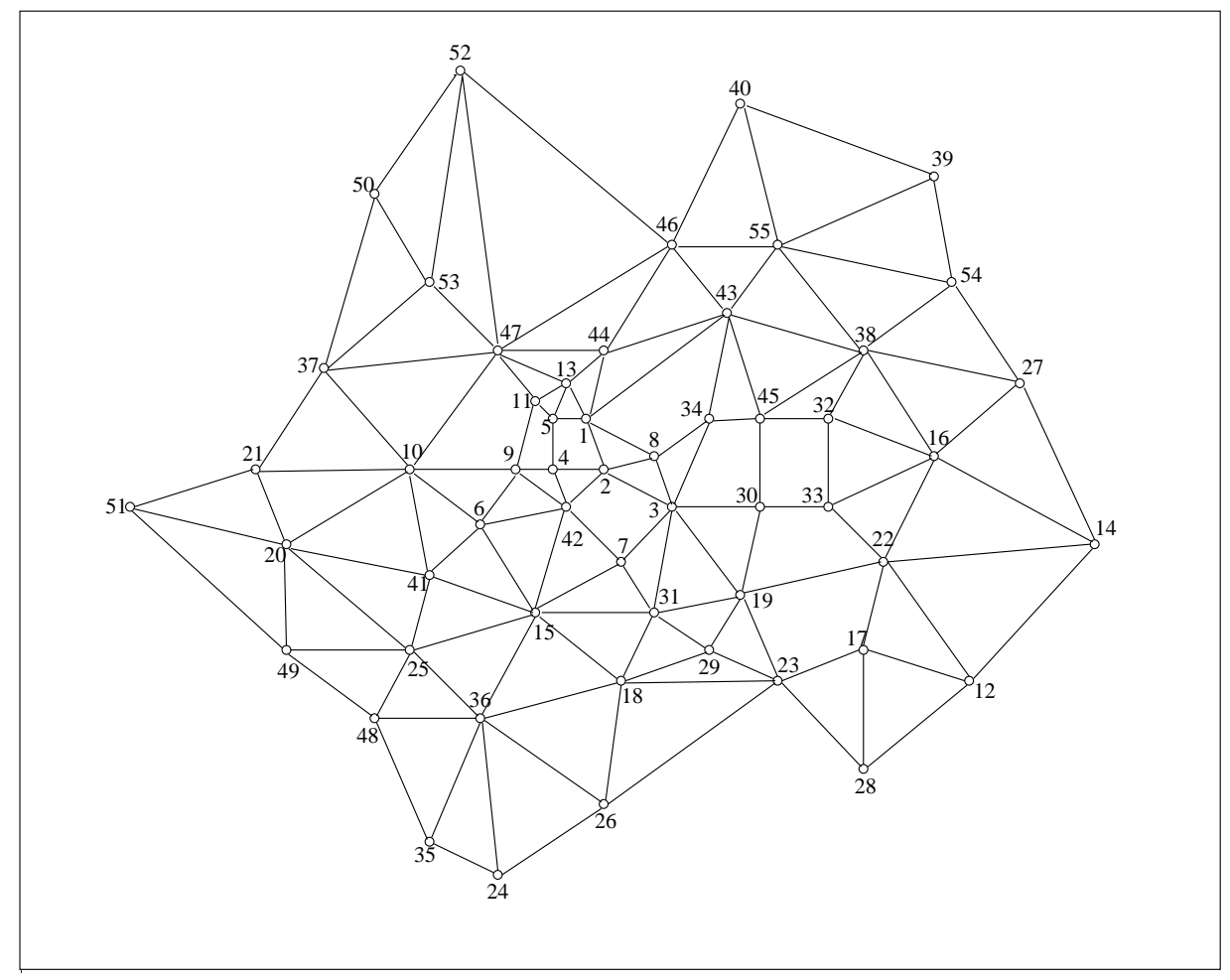

Figure 1: 55-node network 
Table 1

\begin{tabular}{|c|c|c|c|c|c|}
\hline \multirow[t]{2}{*}{$\left(n^{A}, n^{B}\right)$} & \multirow[t]{2}{*}{$\alpha$} & \multicolumn{3}{|c|}{$\%$ of optimal solutions found } & non-opt. \\
\hline & & Phase I & Phase II & Phase III & solutions \\
\hline \multirow[t]{3}{*}{$(2,2)$} & 0.5 & 0 & $90 \%$ & $7 \%$ & $3 \%$ \\
\hline & 1.0 & 0 & $95 \%$ & $3 \%$ & $2 \%$ \\
\hline & 1.5 & 0 & $96 \%$ & $2 \%$ & $2 \%$ \\
\hline \multirow[t]{3}{*}{$(3,3)$} & 0.5 & 0 & $75 \%$ & $15 \%$ & $10 \%$ \\
\hline & 1.0 & 0 & $81 \%$ & $15 \%$ & $4 \%$ \\
\hline & 1.5 & 0 & $92 \%$ & $4 \%$ & $4 \%$ \\
\hline
\end{tabular}

Table 2

\begin{tabular}{|c|c|ccc|}
\hline & & \multicolumn{3}{|c|}{} \\
\hline & $\left(n^{A}, n^{B}\right)$ & $\alpha$ & \multicolumn{3}{|c|}{ Average Computer time (seconds) } \\
\hline & & Phase I & Phase II & Phase III \\
\hline \multirow{3}{*}{$(2,2)$} & 0.5 & 0.27 & 20.35 & 139.98 \\
& 1.0 & 0.24 & 8.89 & 54.22 \\
& 1.5 & 0.11 & 5.64 & 35.12 \\
\hline \multirow{3}{*}{$(3,3)$} & 0.5 & 0.34 & 44.27 & 218.14 \\
& 1.0 & 0.11 & 20.21 & 93.76 \\
& 1.5 & 0.24 & 9.56 & 63.02 \\
\hline
\end{tabular}


Table 3: Results, 55-node network

\begin{tabular}{|c|c|c|c|c|c|}
\hline & \multirow{2}{*}{$\begin{array}{l}\text { Firm B's } \\
\text { Monopoly }\end{array}$} & \multicolumn{3}{|c|}{ Firm A's results } \\
\hline & & & Phase 1 & Phase 2 & Phase 3 \\
\hline $\begin{array}{c}\alpha=0.3 \\
(3,3)\end{array}$ & $\begin{array}{l}\Pi^{A} \\
\Pi^{B} \\
p^{A} \\
p^{B} \\
J^{A} \\
J^{B}\end{array}$ & $\begin{array}{c}107,414 \\
119.5 \\
4,22,31\end{array}$ & $\begin{array}{c}41,113 \\
40,720 \\
40.0 \\
60.0 \\
2,9,16 \\
4,22,31\end{array}$ & $\begin{array}{c}53,460 \\
55,661 \\
65.0 \\
86.5 \\
5,17,41 \\
4,22,31\end{array}$ & $\begin{array}{c}53,460 \\
55,661 \\
65.0 \\
86.5 \\
5,17,41 \\
4,22,31\end{array}$ \\
\hline $\begin{array}{c}\alpha=1.0 \\
(3,3)\end{array}$ & $\begin{array}{l}\Pi^{A} \\
\Pi^{B} \\
p^{A} \\
p^{B} \\
J^{A} \\
J^{B}\end{array}$ & $\begin{array}{r}17,948 \\
40.5 \\
3,5,9\end{array}$ & $\begin{array}{c}8,673 \\
12,324 \\
29,5 \\
40,5 \\
1,6,30 \\
3,5,9\end{array}$ & $\begin{array}{c}9,974 \\
11,633 \\
30.5 \\
40,5 \\
1,6,7 \\
3,5,9\end{array}$ & $\begin{array}{c}9,974 \\
11,633 \\
30.5 \\
40,5 \\
1,6,7 \\
3,5,9\end{array}$ \\
\hline $\begin{array}{c}\alpha=0.3 \\
(5,5)\end{array}$ & $\begin{array}{l}\Pi^{A} \\
\Pi^{B} \\
p^{A} \\
p^{B} \\
J^{A} \\
J^{B}\end{array}$ & $\begin{array}{c}122,577 \\
114.8 \\
5,20,22,24,31\end{array}$ & $\begin{array}{c}48,351 \\
35,606 \\
35.0 \\
52.5 \\
4,13,17,18,34 \\
5,20,22,24,31 \\
\end{array}$ & $\begin{array}{c}68,881 \\
58,583 \\
64.5 \\
93.0 \\
4,16,17,30,41 \\
5,20,22,24,31\end{array}$ & $\begin{array}{c}68,881 \\
58,583 \\
64.5 \\
93.0 \\
4,16,17,30,41 \\
5,20,22,24,31\end{array}$ \\
\hline $\begin{array}{c}\alpha=1.0 \\
(5,5)\end{array}$ & $\begin{array}{l}\Pi^{A} \\
\Pi^{B} \\
p^{A} \\
p^{B} \\
J^{A} \\
J^{B}\end{array}$ & $\begin{array}{c}22,282 \\
43.0\end{array}$ & $\begin{array}{c}10,734 \\
13,740 \\
21.0 \\
43.0 \\
4,13,15,21,30 \\
5,7,8,9,10\end{array}$ & $\begin{array}{c}13,760 \\
14,260 \\
43.0 \\
43.0 \\
1,2,3,6,11 \\
5,7,8,9,10\end{array}$ & $\begin{array}{c}13,760 \\
14,260 \\
43.0 \\
43.0 \\
1,2,3,6,11 \\
5,7,8,9,10\end{array}$ \\
\hline
\end{tabular}


Table 4: 55-node Network demand intercepts and coordinates

\begin{tabular}{||c||r|r||r|r|r||r|r|r||}
\hline \hline \multicolumn{1}{||c||}{ node } & \multicolumn{2}{c|}{ coord } & node & \multicolumn{2}{c|}{ coord } & node & \multicolumn{2}{c||}{ coord } \\
\hline 1 & 32 & 31 & 20 & 25 & 14 & 39 & 46 & 51 \\
2 & 29 & 32 & 21 & 29 & 12 & 40 & 50 & 40 \\
3 & 27 & 36 & 22 & 24 & 48 & 41 & 23 & 22 \\
4 & 29 & 29 & 23 & 17 & 42 & 42 & 27 & 30 \\
5 & 32 & 29 & 24 & 6 & 26 & 43 & 38 & 39 \\
6 & 26 & 25 & 25 & 19 & 21 & 44 & 36 & 32 \\
7 & 24 & 33 & 26 & 10 & 32 & 45 & 32 & 41 \\
8 & 30 & 35 & 27 & 34 & 56 & 46 & 42 & 36 \\
9 & 29 & 27 & 28 & 12 & 47 & 47 & 36 & 26 \\
10 & 29 & 21 & 29 & 19 & 38 & 48 & 15 & 19 \\
11 & 33 & 28 & 30 & 27 & 41 & 49 & 19 & 14 \\
12 & 17 & 53 & 31 & 21 & 35 & 50 & 45 & 19 \\
13 & 34 & 30 & 32 & 32 & 45 & 51 & 27 & 5 \\
14 & 25 & 60 & 33 & 27 & 45 & 52 & 52 & 24 \\
15 & 21 & 28 & 34 & 32 & 38 & 53 & 40 & 22 \\
16 & 30 & 51 & 35 & 8 & 22 & 54 & 40 & 52 \\
17 & 19 & 47 & 36 & 15 & 25 & 55 & 42 & 42 \\
18 & 17 & 33 & 37 & 35 & 16 & & & \\
19 & 22 & 40 & 38 & 36 & 47 & & & \\
\hline \hline
\end{tabular}

